\title{
Time-encoded mid-infrared Fourier-domain optical coherence tomography
}

\author{
Ivan Zorin ${ }^{1, *}$, Paul Gattinger ${ }^{1}$, Andrii Prylepa ${ }^{1}$, Bettina Heise ${ }^{1}$ \\ Research Center for Non-Destructive Testing, Science Park 2, \\ Altenberger Str.69, 4040 Linz, Austria \\ September 15, 2021 \\ *Corresponding author: ivan.zorin@ recendt.at \\ Accepted manuscript; Ivan Zorin, Paul Gattinger, Andrii Prylepa, and Bettina Heise, \\ “Time-encoded mid-infrared Fourier-domain optical coherence tomography," Opt. Lett. 46, (2021). \\ https://doi.org/10.1364/OL.434855 \\ (C) 2021 Optical Society of America
}

\begin{abstract}
We report on a technically simple approach to achieve high-resolution and high-sensitivity Fourier-domain OCT imaging in the mid-infrared range. The proposed OCT system employs an $\mathrm{InF}_{3}$ supercontinuum source. A specially designed dispersive scanning spectrometer based on a single InAsSb point detector is employed for detection. The spectrometer enables structural OCT imaging in the spectral range from $3140 \mathrm{~nm}$ to $4190 \mathrm{~nm}$ with a characteristic sensitivity of over $80 \mathrm{~dB}$ and an axial resolution below $8 \mu \mathrm{m}$. The capabilities of the system are demonstrated for imaging of porous ceramic samples and transition-stage green parts fabricated using an emerging method of lithography-based ceramic manufacturing. Additionally, we demonstrate the performance and flexibility of the system by OCT imaging using an inexpensive low-power (average power of $16 \mathrm{~mW}$ above $3 \mu \mathrm{m}$ wavelength) mid-IR supercontinuum source.
\end{abstract}

Optical coherence tomography (OCT) is a wellestablished non-invasive and non-contact method for threedimensional structural imaging of complex specimens. OCT is of major importance and is primarily used in biomedical scenarios and ophthalmology [1]. In the last few years, prospects and effectiveness of OCT beyond biomedicine [2] have markedly increased in terms of applied research, as the technique has been pushed to longer wavelengths - first, to the border of the near-infrared (nearIR) range [3, 4], then further into the mid-IR region. The reports on mid-IR OCT (either implemented in direct sens- ing mode based on thermal detection [5, 6] or employing nonlinear conversion techniques [7, 8]) have demonstrated advanced capabilities of long wavelengths for probing scattering media. Since the magnitude of scattering decreases with increasing wavelength, it has become possible to examine samples previously ineligible for testing. In this way, these reports have demonstrated the benefits of shifting to the mid-IR spectral region and have identified application opportunities, but also challenges.

As a result, mid-IR OCT has become an emerging subfield that has already gained significant attention in the field of non-destructive testing and defectoscopy of strongly scattering industrial samples (with no water content, due to strong water absorption in this range). The main obstacle that still severely limits the effectiveness and widespread adoption of OCT operating in this spectral band is essentially technical. It is caused by lagging mid-IR detection technologies or their technical complexity. It is particularly interesting that the presented mid-IR OCT systems demonstrated enhanced probing capabilities despite severely limited sensitivities (from approximately $60 \mathrm{~dB}$ to $80 \mathrm{~dB}$ ) compared to well-developed near-IR OCT counterparts. Thus, the primary reason was to establish mid-IR OCT in the Fourier-domain (FD-OCT) configuration that has a fundamental sensitivity advantage over the time-domain OCT (TD-OCT) [9, 10]. In the former case, all depth components are captured simultaneously and do not contribute to depth-dependent noise as inherent in TD-OCT.

The FD-OCT configuration poses a number of realization complications, when transferring to the mid-IR range (mid-IR swept-sources are not yet technically mature for OCT). For example, unlike TD-OCT, which uses a single- 
point detector, the FD-OCT requires a broadband mid-IR spectrometer with well-separated spectral channels, based on a linear array in the standard configuration. State-of-theart mid-IR focal plane arrays exhibit compromised sensitivity (inherent for low bandgap semiconductors as well as for thermal detectors). These arrays are expensive (in particular photonic systems) and have small numbers of pixels (thermal systems). For this reason, the reported midIR OCT realizations either exploited nonlinear conversion techniques to perform detection using well-developed nearIR technologies, or employed direct sensing using a pyroelectric array with proper handling of the resulting limitations. In the first case, the sensitivity of the systems is strongly affected by the relative inefficiency of the conversion techniques. However, broad spectral ranges and highperformance cameras enabled a high axial resolution and a reasonable sensitivity roll-off. In contrast, direct sensing OCT based on thermal arrays offered higher sensitivity, but had lower axial resolution and a strong trade-off between spatial performance and signal roll-off. In this case, the spectral range could not be broadened, which led to a sharp drop of sensitivity over depth, as the spectral resolution of the spectrometer is severely limited by the number of pixels.

In this letter, we address these issues and report on a new approach of mid-IR time-encoded FD-OCT (teFDOCT) [11, 12] that not only provides high sensitivity and axial resolution, but is also technically simple and costeffective. We present a detection system well suited for mid-IR OCT in the current state of the detection technology. This solution elegantly combines the advantages of TD- and FD-OCT by using a single-point detector whilst achieving the sensitivity advantage of FD-OCT [12].

The basic optical layout and operating principles of the mid-IR teFD-OCT imager are shown in Fig. 1. The system is free-space, based on a Michelson interferometer [see Fig. [1.a)] that is formed by a pellicle beamsplitter (nitrocellulose membrane-based, BP145B4, Thorlabs). A 1-inch gold flat mirror is employed in the reference arm. In the sample arm of the interferometer, the supercontinuum beam is focused onto the sample (fixed on a three-axis motorized stage) using a standard $\mathrm{BaF}_{2}$ singlet lens (6 mm thick, antireflection coated, $50 \mathrm{~mm}$ focal length, no strong influence of chromatic aberration observed). Due to the significantly wider operational spectral bandwidth accessed by the developed spectrometer, an effect of group velocity dispersion degrading the axial resolution was clearly observed. The lens is a singlet, thus, correction of the sample arm dispersion was done by inserting $\mathrm{a} \mathrm{BaF}_{2}$ flat window $(5 \mathrm{~mm}$ thick, anti-reflection coated) in the reference arm. The scheme of the interferometer and focusing optics has not changed from the one demonstrated in [13], hence the characterization of lateral resolution (39 $\mu \mathrm{m}$ at $4 \mu \mathrm{m}$ wavelength) is not given here.

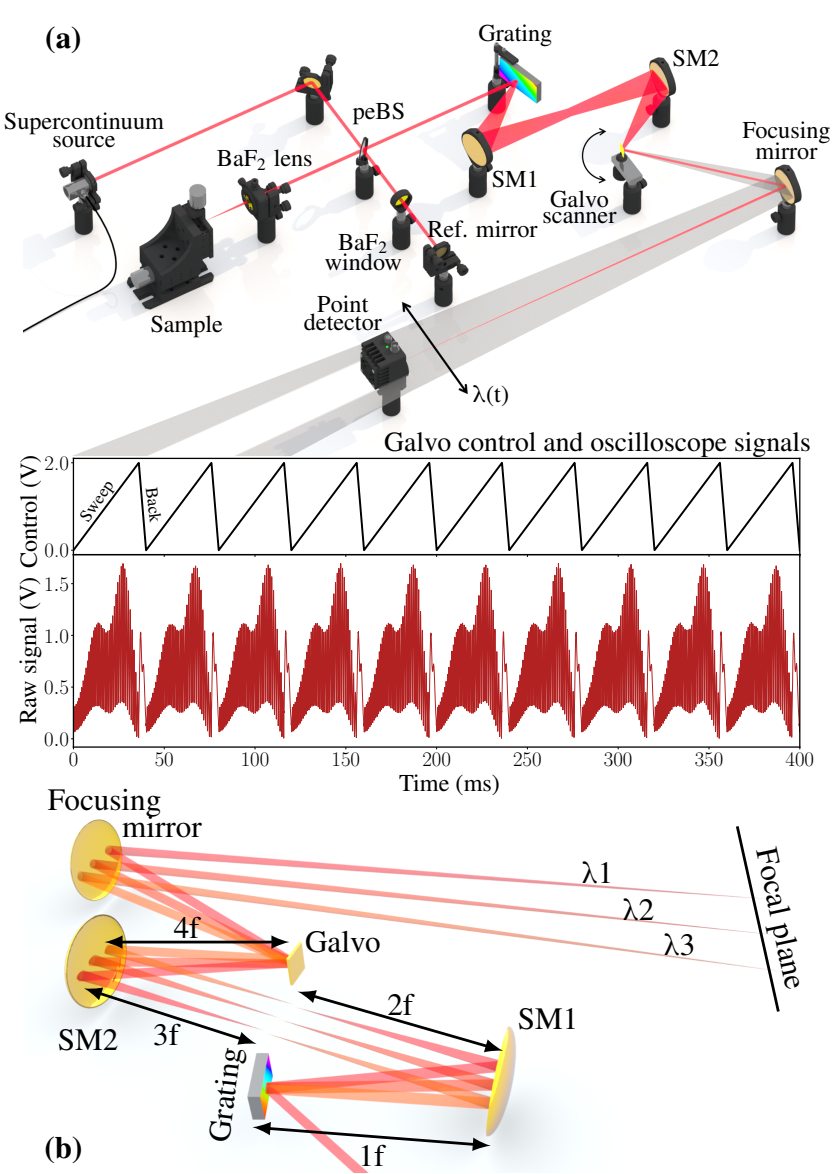

Figure 1: Basic scheme (simplified) and operational principles of the mid-IR teFD-OCT; (a) optical layout of the OCT system, peBS - pellicle beam splitter; SM1 and SM2 - identical spherical mirrors forming a $4 \mathrm{f}$ system to conjugate the grating and scanning planes; raw asymmetric galvo control signals and corresponding real measured spectral interferograms (for a $16 \mathrm{~mW}$ mid-IR supercontinuum source) are shown below; (b) detailed schematic optical diagram of the spectrometer.

For the principal design of the key assembly, i.e. the dispersive scanning OCT spectrometer, we adopted and customized the concept of a high-finesse polygon-based wavelength-scanning filter proposed in [14, 15, 16]. Besides, various aspects of the employed approach have been also intensively considered and discussed in [17, 18]. (quite a similar idea was introduced in [19]) In order to make the system compatible and effective for mid-infrared OCT imaging, the refractive optics constituting the $4 \mathrm{f}$ system in the original design was replaced with reflective optics. This change was motivated by the fact that mirrors are free from chromatic aberration, which can dominate for the broad spectral range of the mid-IR OCT, disturbing performances 
of the spectrometer. Achromatic lenses in the mid-IR spectral region are still limited in their performances - either in spectral bandwidth, efficiency or in aperture sizes (apertures above 1-inch are required). Hence, we employed standard spherical concave mirrors (gold, $100 \mathrm{~mm}$ focal length, tilted by $8^{\circ}$, SM1 and SM2 in Fig. (1) to form the $4 \mathrm{f}$ system that conjugates (in the sagittal plane, where diffraction occurs) the plane of the reflective diffraction grating (300 lines $/ \mathrm{mm}$ ) and the scanner. Instead of a polygonal scanning mirror, we utilized a galvo scanner (at scanning rates from 25 up to $60 \mathrm{~Hz}$, i.e. typical A-scan rates). This was done to enhance spectral resolution and reduce potential nonlinearities. In the polygonal mirror design, the rotational axis is off the facets, thus, introducing jittering (beam transitions, displacements) in the sagittal plane (i.e. spectral discrimination plane) during scanning. In our design, the rotational motion of the galvo mirror (conjugated to the grating, i.e. different spectral components hit the scanner at different angles and overlap in the plane of the scanning mirror) induces a linear spatial sweep of spectral-angular components. In other words, the scanner selects a certain wavelength over the diffraction angles by deflecting it in a specified direction. The detailed schematic drawing of the teFD-OCT system is shown in Fig. 11.b).

Since the spherical mirrors are used at non-normal incidence, the system exhibits astigmatism. The effects of astigmatism do not introduce any reduction of spectral resolution as they enlarge the spot size in the plane orthogonal to the scanning plane. However, they forced us to change the concept of the tunable system (initially used at the light source side to establish a swept-source using an instantaneous broadband emitter). Hence, we utilized the spectral scanner after the OCT interferometer, i.e. as a detection system. In our configuration, each spectral component selected by the scanner is focused onto a high-sensitivity single point detector (uncooled amplified InAsSb detector; Thorlabs, PDA07P2; $1.0 \times 10^{-10} \mathrm{~W} / \mathrm{Hz}^{1 / 2}$ noise equivalent power) behind a slit (400 $\mu \mathrm{m}$ width, for scattering samples). For focusing, a long focal length spherical mirror (gold, $750 \mathrm{~mm}$ focal length, $200 \mathrm{~mm}$ away from the galvo-scanner, tilted by $19^{\circ}$ ) was used. The scanning spectrometer was designed, optimized and characterized in an optical design software (Zemax). For the given configuration of the focusing optics, beam size and the slit width, the spectral resolution of the spectrometer is $1.9 \mathrm{~nm}$ (with no slit inserted i.e., for the entire detector size of $0.7 \mathrm{~mm}$, the resolution is $3.3 \mathrm{~nm}$ ). In summary, the developed spectrometer allowed us to simply implement a virtual high-resolution and highsensitivity mid-IR linear array (equivalent physical arrays are unavailable for this spectral region) with time-separated pixels (as opposed to spatially-separated pixels of cameras).

For experimental demonstrations, primarily a highpower mid-IR supercontinuum source based on $\mathrm{InF}_{3}$ fibre
(Leukos) was used. This source radiates with an average optical power of approximately $230 \mathrm{~mW}$ above $2.4 \mu \mathrm{m}$ wavelength (spanning approximately $4.6 \mu \mathrm{m}$ ); the diameter of the outgoing beam is around $5 \mathrm{~mm}$, the $\mathrm{M}^{2}$ beam quality is 1.13 . The emission is pulsed (ns regime) and features a low dutycycle. The pulse repetition rate is $250 \mathrm{kHz}$, for this reason, a boxcar gated integrator ( 8 cycles averaging for $25 \mathrm{~Hz}$ scanning rate, 4 cycles averaging for $60 \mathrm{~Hz}$ rate) was used to demodulate spectral interferograms and remove inter-pulse noises. An oscilloscope (Red Pitaya, STEMlab 125-14) was used to acquire signals. To illustrate the flexibility and capabilities of the developed teFD-OCT system, we also utilized a ZBLAN fiber-based supercontinuum emitter (test system, NKT Photonics), radiating only $16 \mathrm{~mW}$ of average optical power above $3 \mu \mathrm{m}$ wavelength at $40 \mathrm{kHz}$ repetition rate (sub-ns regime, $2.7 \mathrm{~mm}$ beam diameter, $\mathrm{M}^{2}=1.09$ ). Due to the pronounced spectral non-uniformity, a spectral bandwidth of approximately $3.4 \mu \mathrm{m}$ to $4.1 \mu \mathrm{m}$ was effectively used in this case.

The operating spectral band of the scanning spectrometer was calibrated prior to the remapping of spectral interferograms into $k$-space (the same algorithm as in [5] was used). For this purpose, tools provided by mid-IR spectroscopy were applied. Thus, sharp absorption peaks of polyethylene terephthalate (PET) caused by $\mathrm{CH}$ (carbon-hydrogen) bond stretching vibrations and absorption bands of carbon dioxide $\left(\mathrm{CO}_{2}\right)$ in air were used as spectral features to get a calibration vector. Reference spectral measurements were performed with a commercial high-precision Fouriertransform infrared spectrometer (FTIR, Vertex 70, Bruker Optics). The calibration function appeared to be nonlinear, presumably due to the varying speed of the scanner at the beginning and end of a sweep cycle. However, the recorded spectra and fringes (phase and frequency) are highly timerepeatable and instabilities are not noticeable (i.e. nonlinearity does not fluctuate from sweep to sweep). Hence, the calibration procedure has been performed once and the calibration vector has been stored. The calibrated spectral window accessed by the spectrometer thus ranges from $3138 \mathrm{~nm}$ to $4189 \mathrm{~nm}$. Figure 2(a) depicts details on the calibration procedure and spectral features used, raw spectra of the PET film (also exhibit interference patterns produced by double reflections) and background spectra (sources emissions) are shown. The measurements using the OCT spectrometer were performed with the film inserted in the reference arm, the sample arm was blocked. The calibrated emission spectrum of the $\mathrm{InF}_{3}$ supercontinuum source used for OCT imaging and a typical spectral interferogram (for a single reflector) are shown in Fig. 2. b).

In addition to the hardware dispersion compensation, residual dispersion effects and nonlinearities were compensated numerically using a straightforward amplitude-based approach [20]. Assuming prior physical knowledge of an 


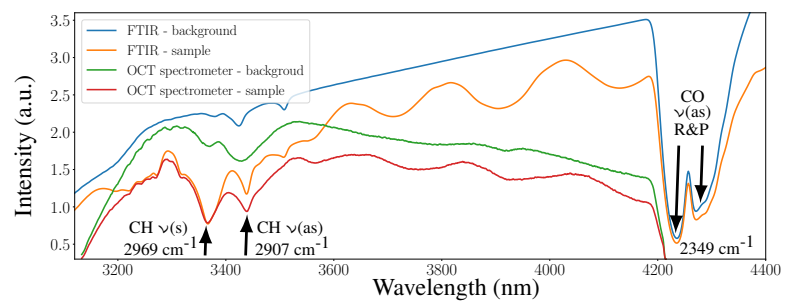

(a) Spectroscopic calibration of the scanning OCT spectrometer (teSystem) using absorption bands of a PET polymer film and $\mathrm{CO}_{2}$ as a reference (FTIR measurements); for the sake of clarity, raw spectra shown (spectral features used for calibration highlighted)

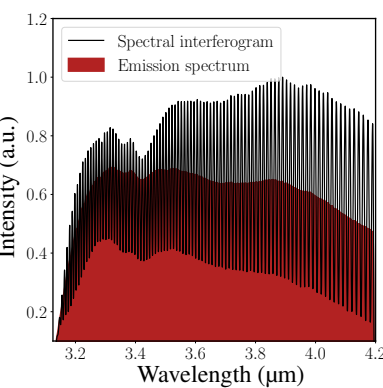

(b) Spectrum of the $\mathrm{InF}_{3}$ supercontinuum source used in OCT imaging; calibrated range $(3138 \mathrm{~nm}-4189 \mathrm{~nm})$

Figure 2: Performances of the teFD-OCT system: (a) calibration of the operational band; (b) accessed spectral bandwidth of mid-IR OCT (emission of the $\mathrm{InF}_{3}$ fibre-based supercontinuum [Leukos]); (c) corresponding axial point spread functions.

ideal OCT signal that the fringes are equally spaced, we exploited peak positions of a reference spectral interferogram recorded for a single interface to eliminate the residual local nonlinear behaviour (similar to a zero-crossing method). The derived calibration vector was implemented into the post-processing algorithm for correction of signals in $k$-space. Figure 2(c) shows an obtained axial point spread function and characterization of the axial resolution $(7.6 \mu \mathrm{m})$. The sensitivity of the system based on the $\mathrm{InF}_{3}$ supercontinuum source was experimentally characterized to be $80.17 \mathrm{~dB}$ (at $25 \mathrm{~Hz}$ scanning rate), using the method described in [21]. The teFD-OCT system based on the lowpower ZBLAN source has a sensitivity of $69.3 \mathrm{~dB}$ and an axial resolution of $16 \mu \mathrm{m}$.

The performance of the developed mid-IR OCT system was validated for structural imaging of porous industrial samples produced by an additive method of lithographybased ceramic manufacturing (LCM). Figure 3 depicts typical OCT scans of sintered highly scattering ceramic plates (approx. $325 \mu \mathrm{m}$ and $480 \mu \mathrm{m}$ thick). The system demon- strated enhanced probing capabilities that are common for mid-IR OCT (the morphology could not be accessed by commercial near-IR OCT systems). The back interfaces of the test samples were revealed and a production curvature defect in the thin plate $(325 \mu \mathrm{m})$ was observed. In
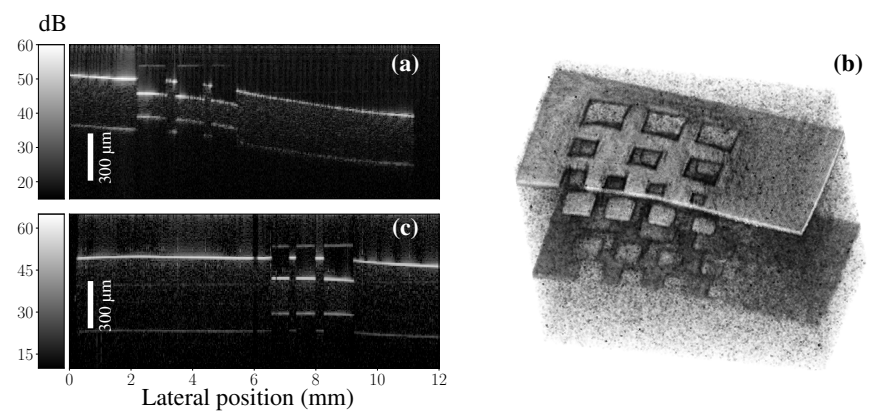

Figure 3: OCT imaging of strongly scattering (alumina, 1$2 \%$ porosity) ceramic micro-structured test-plates: $325 \mu \mathrm{m}$ thick sample [(a) B-scan (12 sec measurement time), (b) volumetric scan (20 min measurement time); and (c) B-scan of $480 \mu \mathrm{m}$ thick plate (12 sec measurement time).

addition, samples showing a different class of LCM defects were examined. Figure 4 shows mid-IR OCT imaging of alumina ceramics (same material properties) with local subsurface porosity variations. The presence of short mid-IR wavelengths in the broad OCT bandwidth allowed us to gain some advantage and visualize varying scattering behaviour. Thus, depth profiles of clusters with reduced porosity were accessed. High porosity zones surrounding the clusters could be identified.
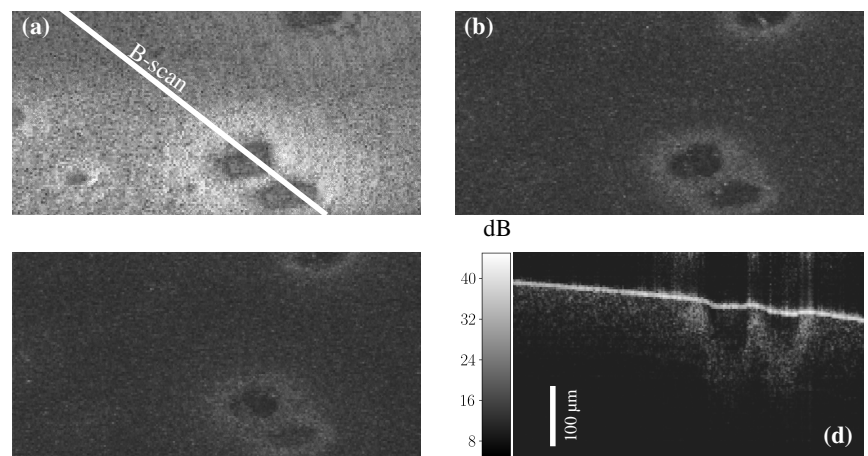

Figure 4: Sub-surface porosity heterogeneities for LCMprinted ceramics: (a-c) en-face images $\left(10 \times 5 \mathrm{~mm}^{2}\right.$ area) at different depths (surface, about $100 \mu \mathrm{m}, 150 \mu \mathrm{m},(25 \mathrm{~min}$ measurement time)); (d) B-scan, the effective depth is limited to around $0.4 \mathrm{~mm}$ for the alumina group index (1.83).

Particularly interesting results were obtained for imaging of green LCM parts (intermediate production stage) using a $16 \mathrm{~mW}$ mid-IR supercontinuum source. The samples are 
strongly scattering, and made of a solid, flexible material that consists of ceramic particles embedded in a polymer matrix (on the basis of acrylate and methacrylate). A Bscan of the $650 \mu \mathrm{m}$ thick green sample [corresponds to the sintered part shown in Fig. 3 (b)] is depicted in Fig. 5[a). For comparison, a B-scan obtained using a state-of-the art commercial near-IR OCT $(1.3 \mu \mathrm{m}$ center wavelength, sweptsource OCT, Vega, Thorlabs) is shown in Fig. 5(b). The recorded scans demonstrate the enhanced probing performance but also perspectives for the mid-IR OCT based on the low-power light source; for the near-IR OCT system multiple scattering dominates, the bottom interface is not detected.
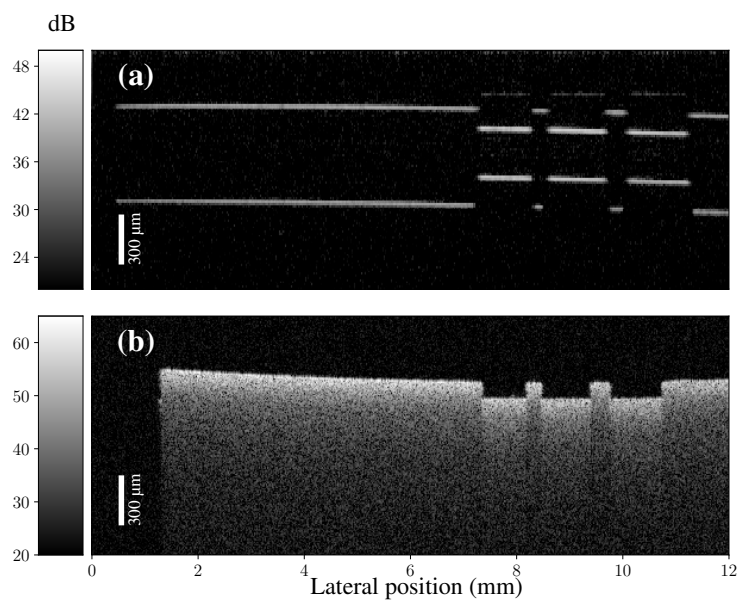

Figure 5: OCT imaging of the green LCM parts using (a) the mid-IR teFD-OCT with the $16 \mathrm{~mW}$ supercontinuum source (15 sec measurement time) and (b) the commercial $1.3 \mu \mathrm{m}$ SS-OCT system.

In conclusion, we have proposed and experimentally demonstrated a concept of mid-IR time-encoded FD-OCT operating in direct sensing mode. The developed system combines the strengths of the TD- and FD-OCT configurations as it employs a single pixel detector, but also inherits the specific sensitivity advantage by exploiting the concept of a virtual array with time-separated pixels. The experimental results have indicated practical prospects for applications in field conditions. As future improvements, we foresee the optimization of imaging speed and sensitivity. We expect further adaptation of the optical scheme to enhance spectral performances and see a possibility to establish kinetic mid-IR sweep OCT sources based on a similar design.

Funding Österreichische Forschungsförderungsgesellschaft (FFG) (877481, 856896); State of Upper Austria (Wi-2020-700476/3).

Acknowledgments The authors thank Guillaume Huss from Leukos and Patrick Bowen from NKT Photonics for providing supercontinuum sources; and Martin Schwentenwein and Dominik Brouczek from Lithoz for designing and providing us with various LCM ceramic samples.

\section{References}

[1] J. Fujimoto and E. Swanson, "The Development, Commercialization, and Impact of Optical Coherence Tomography," Investigative Opthalmology \& Visual Science, vol. 57, pp. 1-13, July 2016.

[2] D. Stifter, "Beyond biomedicine: a review of alternative applications and developments for optical coherence tomography," Applied Physics B, vol. 88, pp. 337-357, Aug 2007.

[3] C. S. Cheung, J. M. O. Daniel, M. Tokurakawa, W. A. Clarkson, and H. Liang, "Optical coherence tomography in the $2-\mu \mathrm{m}$ wavelength regime for paint and other high opacity materials," Opt. Lett., vol. 39, pp. 65096512, Nov 2014.

[4] C. S. Cheung, J. M. O. Daniel, M. Tokurakawa, W. A. Clarkson, and H. Liang, "High resolution fourier domain optical coherence tomography in the $2 \mu \mathrm{m}$ wavelength range using a broadband supercontinuum source," Opt. Express, vol. 23, pp. 1992-2001, Feb 2015.

[5] I. Zorin, R. Su, A. Prylepa, J. Kilgus, M. Brandstetter, and B. Heise, "Mid-infrared fourier-domain optical coherence tomography with a pyroelectric linear array," Opt. Express, vol. 26, pp. 33428-33439, Dec 2018.

[6] I. Zorin, P. Gattinger, M. Brandstetter, and B. Heise, "Dual-band infrared optical coherence tomography using a single supercontinuum source," Opt. Express, vol. 28, pp. 7858-7874, Mar 2020.

[7] N. M. Israelsen, C. R. Petersen, A. Barh, D. Jain, M. Jensen, G. Hannesschläger, P. TidemandLichtenberg, C. Pedersen, A. Podoleanu, and O. Bang, "Real-time high-resolution mid-infrared optical coherence tomography," Light: Science \& Applications, vol. 8, pp. 2047-7538, Jan 2019.

[8] A. Vanselow, P. Kaufmann, I. Zorin, B. Heise, H. M. Chrzanowski, and S. Ramelow, "Frequency-domain optical coherence tomography with undetected midinfrared photons," Optica, vol. 7, pp. 1729-1736, Dec 2020. 
[9] R. Leitgeb, C. K. Hitzenberger, and A. F. Fercher, "Performance of fourier domain vs. time domain optical coherence tomography," Opt. Express, vol. 11, pp. 889-894, Apr 2003.

[10] M. A. Choma, M. V. Sarunic, C. Yang, and J. A. Izatt, "Sensitivity advantage of swept source and fourier domain optical coherence tomography," Opt. Express, vol. 11, pp. 2183-2189, Sep 2003.

[11] W. Drexler and J. G. Fujimoto, Optical Coherence Tomography, Technology and Applications. Springer International Publishing, 2008.

[12] B. Považay, A. Unterhuber, B. Hermann, H. Sattmann, H. Arthaber, and W. Drexler, "Full-field time-encoded frequency-domain optical coherence tomography," Opt. Express, vol. 14, pp. 7661-7669, Aug 2006.

[13] I. Zorin, R. Su, B. Heise, B. Lendl, and M. Brandstetter, "Correlative infrared optical coherence tomography and hyperspectral chemical imaging," J. Opt. Soc. Am. A, vol. 37, pp. B19-B26, Sep 2020.

[14] S. H. Yun, C. Boudoux, G. J. Tearney, and B. E. Bouma, "High-speed wavelength-swept semiconductor laser with a polygon-scanner-based wavelength filter," Opt. Lett., vol. 28, pp. 1981-1983, Oct 2003.

[15] W. Y. Oh, S. H. Yun, G. J. Tearney, and B. E. Bouma, "115 khz tuning repetition rate ultrahighspeed wavelength-swept semiconductor laser," Opt. Lett., vol. 30, pp. 3159-3161, Dec 2005.

[16] W.-Y. Oh, B. J. Vakoc, M. Shishkov, G. J. Tearney, and B. E. Bouma, "¿400 khz repetition rate wavelength-swept laser and application to high-speed optical frequency domain imaging," Opt. Lett., vol. 35, pp. 2919-2921, Sep 2010.

[17] M. Everson, V.-F. Duma, and G. Dobre, "Aspects of vignetting in a polygon mirror-based spectral filter for swept source optical coherence tomography (SSOCT)," in Seventh International Conference on Lasers in Medicine (C. T. M.D., A. Podoleanu, and V.-F. Duma, eds.), vol. 10831, pp. 98 - 106, International Society for Optics and Photonics, SPIE, 2018.

[18] M. Everson, V.-F. Duma, and G. Dobre, "Optimisation of a polygon mirror-based spectral filter for swept source optical coherence tomography (SS-OCT)," in 2nd Canterbury Conference on OCT with Emphasis on Broadband Optical Sources (A. Podoleanu and O. Bang, eds.), vol. 10591, pp. 180 - 186, International Society for Optics and Photonics, SPIE, 2018.
[19] A. L. Oldenburg, J. J. Reynolds, D. L. Marks, and S. A. Boppart, "Fast-fourier-domain delay line for in vivo optical coherence tomography with a polygonal scanner," Appl. Opt., vol. 42, pp. 4606-4611, Aug 2003.

[20] M. Duelk and K. Hsu, "SLEDs and Swept Source Laser Technology for OCT," in Optical Coherence Tomography (W. Drexler and J. G. Fujimoto, eds.), pp. 527-561, Cham: Springer International Publishing, 2015.

[21] A. Agrawal, T. J. Pfefer, P. D. Woolliams, P. H. Tomlins, and G. Nehmetallah, "Methods to assess sensitivity of optical coherence tomography systems," Biomed. Opt. Express, vol. 8, pp. 902-917, Feb 2017. 